\title{
Coloração do Fruto e Substrato na Emergência e no Crescimento de Plantas de Eugenia calycina Cambess
}

\author{
Kelly Cristiene de Freitas Borges ${ }^{1}$, Denise Garcia de Santana ${ }^{2}$, \\ Susana Webber Lopes ${ }^{2}$, Vanderley José Pereira ${ }^{2}$ \\ ${ }^{1}$ Instituto de Ciências Agrárias, Universidade Federal de Goiás - UFG, Goiânia/GO, Brasil \\ ${ }^{2}$ Instituto de Ciências Agrárias, Universidade Federal de Uberlândia - UFU, Uberlândia/MG, Brasil
}

\begin{abstract}
RESUMO
O objetivo do trabalho foi avaliar a influência da coloração dos frutos na emergência e no crescimento das plantas de Eugenia calycina Cambess (pitangueira-do-cerrado), assim como de substratos com diferentes proporções de material orgânico e estruturante. Três experimentos foram instalados: o primeiro de emergência; o segundo de crescimento de plantas constituídos de sementes provenientes de frutos verdes, alaranjados/avermelhados, vermelho-claros e vermelho-escuros; e o terceiro comparou cinco misturas de Bioplant ${ }^{\circledR}$ suplementado com Vermiculita ${ }^{\circledR}$ e com pó-de-coco no crescimento das plantas, ambos nas proporções de $20 \%$ e $40 \%$. A coloração do fruto não influenciou a capacidade de emergência, tempo (início, médio e final), velocidade e sincronia de emergência das plântulas de E. calycina. O crescimento das plantas até 270 dias de cultivo foi independente da coloração do fruto. A Vermiculita ${ }^{\circledR}$ e o pó-de-coco suplementados ao Bioplant ${ }^{\circledast}$ não afetaram o crescimento das plantas até os 120 dias de cultivo.
\end{abstract}

Palavras-chave: maturação do fruto, fruteiras nativas, pitangueira-do-cerrado, sementes.

\section{Fruit Coloring and Substrate in Emergence and Growth of Eugenia calycina Cambess Seedlings}

\begin{abstract}
This study aimed to evaluate the influence of fruit coloring in emergence process and growth of Eugenia calycina Cambess seedlings, as well substrates with different proportions of organic and structuring material. Three experiments were conducted: the first of emergence; the second of plant growth, both with fruits of green color, orange/red, light red and dark red; and the third experiment was conducted to compare five mixture of Bioplant ${ }^{\circledR}$ supplemented with Vermiculita ${ }^{\circledR}$ and coconut i powder $\mathrm{n}$ the seedling growth, both in proportion of 20 and $40 \%$. The fruit coloring did not influence the capacity of emergence, time (initial, mean and final), rate and synchrony of emergence of E. calycina seedlings. The growth of E. calycina seedlings is independent of fruit color up to 270 days. The Vermiculita ${ }^{\circledR}$ and -coconut powder supplemented by Bioplant ${ }^{\circledR}$ do not improved the plants growth up to 120 days of cultivation.
\end{abstract}

Keywords: fruit maturation, native fruits, Cerrados's cherry, seeds. 


\section{INTRODUÇÃO}

Com ampla biodiversidade, o cerrado apresenta espécies frutíferas com potencial para cultivo comercial (Pereira \& Pasquelete, 2011) desempenhando papel ecológico primordial nos ecossistemas (Gomes \& Moura, 2010), além de serem importantes na manutenção do recurso genético para fins de melhoramento (Aquino et al., 2007; Conceição \& Aragão, 2010).

Das espécies frutíferas, a família Myrtaceae é uma das mais importantes no Brasil, com destaque para o gênero Eugenia (Landrum \& Kawasaki, 1997). Registrada no cerrado de Goiás, Minas Gerais e Distrito Federal nos tipos fitofisionômicos de campo sujo, cerrado propriamente dito, campo cerrado e transição cerrado-vereda, Eugenia calycina Cambess (pitangueira-do-cerrado) é uma herbácea com altura entre $40 \mathrm{~cm}$ e $60 \mathrm{~cm}$ (Ribeiro \& Rodrigues, 2006; Cielo et al., 2012). Os frutos, alongados e não possuindo os sulcos externos característicos da pitanga-comum (Eugenia uniflora L.), amadurecem entre setembro e dezembro (Pereira \& Pasquelete, 2011), quando adquirem coloração avermelhada.

Entre as restrições para o cultivo de espécies do gênero Eugenia estão a dificuldade de conservação das sementes e de multiplicação das plantas e a variabilidade genética (dificultando a seleção de matrizes) somada a baixa densidade e frequência de indivíduos nas áreas de ocorrência (Delgado \& Barbedo, 2007; Franzon et al., 2010; Sena et al., 2010; Teixeira \& Barbedo, 2012; Scalon et al., 2012; Camilo et al., 2013; Souza et al., 2013; Tonetto et al., 2013). Contudo, o fracionamento das sementes, com possibilidade de formação de mais de uma plântula normal, ampliaram as expectativas de multiplicação (Silva et al., 2005; Teixeira \& Barbedo, 2012). Outra restrição é a baixa longevidade das sementes, causada por sua intolerância à perda de água após dispersão (Delgado \& Barbedo, 2007; Justo et al., 2007; Oro et al., 2012; Scalon et al., 2012).

Pelo potencial comercial, a domesticação das espécies de Eugenia é fator relevante para a pesquisa científica. Substratos compostos por material orgânico e solo, acrescidos de areia, Vermiculita ${ }^{\circledR}$ e outros elementos químicos e estruturantes estimularam o crescimento das plantas de Eugenia dysenterica DC, Eugenia pyriformis Cambess e E. uniflora (Souza et al., 2002; Abreu et al., 2005; Medeiros et al., 2010). Contudo, a distribuição em áreas de cerrado converge para a hipótese de que E. calycina não tolera substratos com alta capacidade de retenção de água, o que limita alguns materiais usualmente utilizados na sua formulação.

A coloração do fruto e suas mudanças são características que permitem inferir sobre a maturação fisiológica de sementes e, consequentemente, sobre a época adequada de colheita. Essa relação foi comprovada com melhor desempenho das sementes quando colhidas de frutos de coloração esverdeada/amarelada e amarelada/alaranjada de E. pyriformis, vermelha-clara de Eugenia involucrata e vermelha de E. uniflora (Ávila et al., 2009; Oro et al., 2012). O objetivo do trabalho foi avaliar a influência da coloração dos frutos na emergência e no crescimento das plantas de Eugenia calycina Cambess (pitangueira-do-cerrado), assim como de substratos com diferentes proporções de material orgânico e estruturante.

\section{MATERIAL E MÉTODOS}

Frutos de E. calycina, entre 12 e 45 por matriz, foram coletados de 26 matrizes em novembro de 2006 na Reserva Ecológica do Clube de Caça e Pesca Itororó, de Uberlândia, $\mathrm{MG}\left(18^{\circ} 55^{\prime} \mathrm{S}\right.$ e $48^{\circ} 17^{\prime} \mathrm{W}$, altitude aproximada de $890 \mathrm{~m}$ ), formada por cerrado propriamente dito, segundo Cardoso \& Lomônaco (2003). O clima é caracterizado por duas estações com diferença nos índices pluviométricos, que oscilam anualmente em torno de $1.550 \mathrm{~mm}$, e temperatura média anual de $22^{\circ} \mathrm{C}$.

Para a coleta dos frutos foram estabelecidos os padrões de coloração verde, alaranjada/avermelhada, vermelha-clara e vermelha-escura, indicando os diferentes estádios de maturação (Figura 1). Os frutos foram colhidos manualmente e transportados em caixas com isolamento térmico de poliestireno expandido (EPS), para evitar a perda de água e a depreciação. Cerca de 2 horas depois da coleta foi realizada a remoção manual da polpa em água corrente, seguida da disposição das sementes sobre papel filtro por 30 minutos, para a retirada do excesso de água.

Os teores de água das sementes foram determinados imediatamente após o beneficiamento à temperatura de $70{ }^{\circ} \mathrm{C}$ e de $105^{\circ} \mathrm{C}$. A escolha da temperatura $70{ }^{\circ} \mathrm{C}$ com pesagens diárias em balança analítica até massa constante, atingida no terceiro dia, foi por cautela 

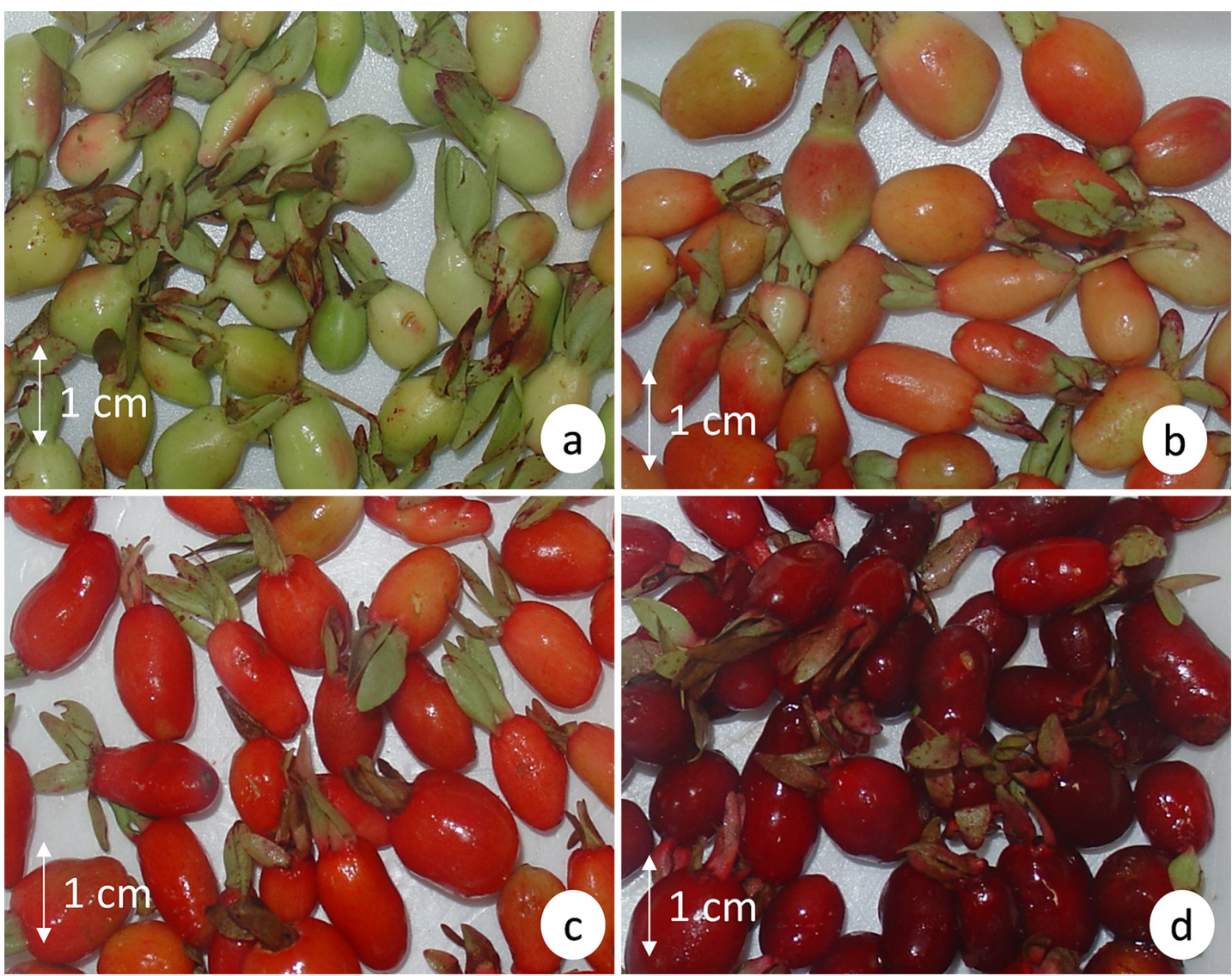

Figura 1. Coloração dos frutos de Eugenia calycina Cambess. (a): verde; (b): alaranjada/avermelhada; (c): vermelha-clara; e (d): vermelha-escura.

Figure 1. Fruit coloring of Eugenia calycina Cambess. (a): green fruit; (b): orange/reddish; (c): light red and (d): dark red.

referente à possível perda de substância voláteis, como sugerido por Borghetti \& Ferreira (2004). A temperatura $103^{\circ} \mathrm{C} \pm 2{ }^{\circ} \mathrm{C}$ por 24 horas, do método conhecido como de estufa a $105^{\circ} \mathrm{C}$, foi utilizada por ser esse o método oficial de determinação (Brasil, 2009).

Para ambas as determinações foram utilizadas 8 repetições de 5 sementes cada. Os teores foram calculados segundo a expressão: $(M M F-M M S)^{\star} 100 / M M F$, em que $M M F$ e $M M S$ são as massas da matéria fresca e matéria seca da semente, respectivamente. Para as comparações do teor de água entre as temperaturas e colorações foram construídos intervalos a 95\% de confiança.

A seguir são descritos os experimentos de emergência (Experimento I) e de crescimento das plântulas provenientes de frutos com diferentes colorações (Experimento II) e em diferentes substratos (Experimento III), o qual avaliou composição e texturas distintas (Figura 2).

Em função da heterogeneidade das condições experimentais, especialmente as relativas à incidência de luz, o experimento de emergência de plântulas (Experimento I; Figura 2) foi instalado em delineamento de blocos casualizados, com 4 tratamentos, correspondentes a sementes provenientes de frutos de coloração: verde, alaranjada/avermelhada, vermelha-clara e vermelha-escura, com 7 repetições de 28 sementes. A semeadura foi realizada a $1 \mathrm{~cm}$ de profundidade, em bandejas de 128 células, com $40 \mathrm{~cm}^{3}$ por célula, contendo substrato Plantmax ${ }^{\circledR}$ e Vermiculita ${ }^{\circledR}$ na proporção 1:1. As bandejas foram levadas para a estufa com $50 \%$ de redução luminosa $\left(31,3 \pm 5,6{ }^{\circ} \mathrm{C}\right.$ e $36,4 \pm 7,8{ }^{\circ} \mathrm{C}$, médias das temperaturas mínima e máxima, respectivamente) e 3 turnos de irrigação por aspersão com duração de 5 minutos. 


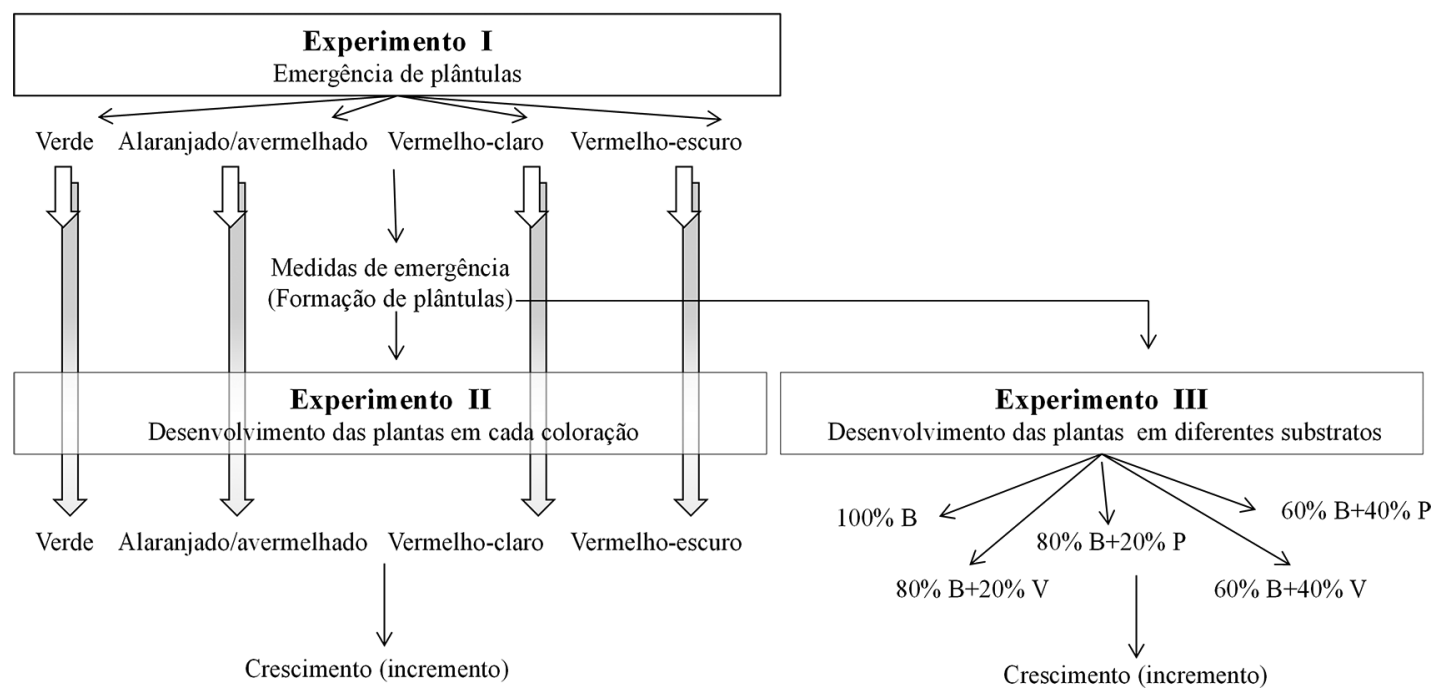

Figura 2. Fluxograma dos experimentos de emergência (Experimento I); crescimento das plantas (Experimento II) advindas dos frutos com as diferentes colorações; e crescimento de plantas em diferentes substratos (Experimento III). B: Bioplant ${ }^{\circledR}$; V: Vermiculita ${ }^{\circledR}$; P: pó-de-coco.

Figure 2. Flowchart of emergence experiments (Experiment I), plant growth (Experiment II) from fruits in different colors and, growth on different substrates (Experiment III). B: Bioplant ${ }^{\circledR}$; V: Vermiculita ${ }^{\circledR}$; P: powder-coconut.

As contagens das plântulas emergidas foram realizadas diariamente, estabilizando-se aos 55 dias após a semeadura. A partir delas foram determinados o percentual de emergência de plântulas, o tempo decorrido da semeadura até a primeira (tempo inicial em dias) e a última emergência (tempo final em dias), o tempo médio de emergência (em dias), a velocidade de emergência, em número de plântulas por dia (Maguire, 1962), e a sincronia (quantidade de sementes que germinam num mesmo intervalo de tempo). Expressões matemáticas, autores e interpretações dessas medidas podem ser consultados em Ranal \& Santana (2006). Foram também calculadas as frequências relativas de emergência, representadas graficamente para cada coloração do fruto.

Para avaliar o crescimento das plantas (Experimento II; Figura 2), conduziu-se um experimento com 7 blocos casualizados, formados por plantas em diferentes fases de desenvolvimento selecionadas do Experimento I. Foram 4 as colorações dos frutos com parcelas formadas por 4 plântulas.

No transplantio foram utilizados tubetes de $180 \mathrm{~cm}^{3}$ contendo substrato Plantmax ${ }^{\circledR} \mathrm{e}$ Vermiculita ${ }^{\circledR}$ na proporção 1:1, acrescido de $5 \mathrm{gL}^{-1}$ de Osmocote ${ }^{\circledast}$, um adubo de liberação lenta (Tabela 1). As bandejas foram levadas para estufa com $50 \%$ de redução luminosa $\left(30,8 \pm 6,6{ }^{\circ} \mathrm{C}\right.$ e $39,4 \pm 5,9^{\circ} \mathrm{C}$, médias das temperaturas mínima e máxima, respectivamente) e 5 turnos de irrigação por aspersão com duração de 7 minutos. As avaliações foram realizadas 210 dias após o transplantio, correspondente a 270 dias após semeadura.

Visando elucidar se a incorporação de material estruturante ao substrato é indispensável ao crescimento das plantas foi instalado o terceiro experimento (III; Figura 2), com 5 misturas. As parcelas foram formadas por 5 plântulas, independente da coloração do fruto, com cerca de $10 \mathrm{~cm}$ de altura e 6 folhas (120 dias após a semeadura), e foram transplantadas para tubetes de $180 \mathrm{~cm}^{3}$ contendo como substrato uma das misturas. As misturas foram: (1) 100\% de Bioplant ${ }^{\circledR}$; (2) $80 \%$ de Bioplant $^{\circledR}+20 \%$ de Vermiculita $^{\circledR}$; (3) $60 \%$ de Bioplant $^{\circledR}+40 \%$ de Vermiculita $^{\circledR}$; (4) 80\% de Bioplant ${ }^{\circledR}$ $+20 \%$ de pó-de-coco; e (5) $60 \%$ de Bioplant ${ }^{\circledR}+40 \%$ de pó-de-coco (Tabela 1). Em todas foram adicionados $5 \mathrm{gL}^{-1}$ de Osmocote ${ }^{\circledast}$.

As bandejas foram dispostas em delineamento de blocos casualizados com 5 repetições em estufa com $50 \%$ de redução luminosa $\left(34,4 \pm 6,1^{\circ} \mathrm{C}\right.$ e $41,5 \pm 8,6^{\circ} \mathrm{C}$, médias das temperaturas mínima e máxima, respectivamente) e 5 turnos de irrigação por aspersão com duração de 


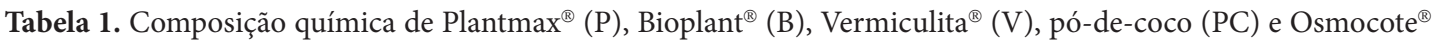
$(\mathrm{O})$.

Table 1. Chemical composition of Plantmax ${ }^{\circledR}(\mathrm{P})$, Bioplant ${ }^{\circledR}(\mathrm{B})$, Vermiculita ${ }^{\circledR}(\mathrm{V})$, powder-coconut $(\mathrm{PC})$ and Osmocote $^{\circledR}(\mathrm{O})$.

\begin{tabular}{|c|c|c|c|c|c|c|c|c|c|c|}
\hline \multirow{2}{*}{${ }^{1}$ Comp. } & \multirow{2}{*}{ Unidade } & \multicolumn{2}{|c|}{ Experimento I } & \multicolumn{3}{|c|}{ Experimento II } & \multicolumn{4}{|c|}{ Experimento III } \\
\hline & & $\mathbf{P}$ & $\mathbf{V}$ & $\mathbf{P}$ & $\mathbf{V}$ & $\mathbf{O}$ & B & V & PC & $\mathbf{0}$ \\
\hline $\mathrm{pH}$ & - & 5,80 & 6,0 & 5,80 & 6,0 & - & 5,2 & 6,00 & 7,5 & - \\
\hline MO & $\%$ & 21,25 & - & 21,25 & - & - & 20,5 & - & 24,77 & - \\
\hline $\mathrm{N}$ & $\%$ & 0,28 & 0,06 & 0,28 & 0,06 & 15,0 & 0,29 & 0,06 & 0,56 & 15,0 \\
\hline $\mathrm{P}$ & $\%$ & 0,13 & 0,02 & 0,13 & 0,02 & 9,0 & 0,74 & 0,02 & 0,92 & 9,0 \\
\hline $\mathrm{K}$ & $\%$ & 0,16 & 0,11 & 0,16 & 0,11 & 12,0 & 0,27 & 0,11 & 0,15 & 12,0 \\
\hline $\mathrm{Ca}$ & $\%$ & 0,55 & 0,07 & 0,55 & 0,07 & - & 0,67 & 0,07 & 0,45 & - \\
\hline $\mathrm{Mg}$ & $\%$ & 0,66 & 11,98 & 0,66 & 11,98 & 1,0 & 0,55 & 11,98 & 0,20 & 1,0 \\
\hline$S$ & $\%$ & 0,07 & 0,14 & 0,07 & 0,14 & 2,30 & 0,35 & 0,14 & 0,02 & 2,3 \\
\hline B & $\mathrm{mg} \mathrm{kg}^{-1}$ & 10,0 & 9,0 & 10,0 & 9,0 & 20,0 & 12,0 & 9,0 & - & 20,0 \\
\hline $\mathrm{Cu}$ & $\mathrm{mg} \mathrm{kg}^{-1}$ & 8,0 & 18,0 & 8,0 & 18,0 & 50,0 & 29,0 & 18,0 & 0,30 & 50,0 \\
\hline $\mathrm{Fe}$ & $\mathrm{mg} \mathrm{kg}^{-1}$ & 8158 & 36185 & 8158 & 36185 & 1000 & 4969 & 36185 & - & 1000 \\
\hline $\mathrm{Mn}$ & $\mathrm{mg} \mathrm{kg}^{-1}$ & 70,0 & 368,0 & 70,0 & 368,0 & 60,0 & 127,0 & 368,0 & 14,0 & 60,0 \\
\hline $\mathrm{Zn}$ & $\mathrm{mg} \mathrm{kg}^{-1}$ & 11,0 & 42,0 & 11,0 & 42,0 & 20,0 & 97,0 & 42,0 & 12,0 & 20,0 \\
\hline $\mathrm{Na}$ & $\mathrm{mg} \mathrm{kg}^{-1}$ & 180,0 & 277,0 & 180,0 & 277,0 & - & 220,0 & 277,0 & - & - \\
\hline
\end{tabular}

${ }^{1}$ Composição: $\mathrm{P}, \mathrm{K}=\left(\mathrm{HCl} \mathrm{0,05} \mathrm{N}+\mathrm{H}_{2} \mathrm{SO}_{4} 0,025 \mathrm{~N}\right) ; \mathrm{Ca}, \mathrm{Mg},(\mathrm{KCl} 1 \mathrm{~N}) ; \mathrm{MO}$ : matéria orgânica (Walkley-Black); $\mathrm{P}$ e K pelo método de extração Mehlich; Ca e Mg, método de extração $\mathrm{KCl} 1 \mathrm{~mol} \mathrm{~L}^{-1}$; Matéria orgânica, método Yeomans e Brenner; $\mathrm{B}=\left[\mathrm{BaCl}_{2} \cdot 2 \mathrm{H}_{2} \mathrm{O}\right.$ a $0,124 \%$ à quente]; $\mathrm{Cu}, \mathrm{Fe}, \mathrm{Mn}, \mathrm{Zn},=[\mathrm{DTPA} 0,005 \mathrm{M}+\mathrm{CaCl} 0,01 \mathrm{M}+\mathrm{TEA} 0,1 \mathrm{M}$ a pH 7,3$] ; \mathrm{S}^{-\mathrm{SO}_{4}}=\mathrm{Ca}_{2}\left(\mathrm{H}_{2} \mathrm{PO}_{4}\right)_{2} 0,01 \mathrm{~mol} \mathrm{~L}^{-1}$.

7 minutos. A avaliação foi realizada aos 60 dias após o transplantio, correspondente a 120 dias da semeadura.

Para os experimentos II e III foram calculados os incrementos em altura $(\mathrm{cm})$, comprimento de raiz $(\mathrm{cm})$, diâmetro do caule ( $\mathrm{cm}$; tomado no ponto de inserção da base entre a parte aérea e o sistema radicular) e número de folhas. As medidas de emergência (Experimento I) e de crescimento (experimentos II e III) foram analisadas utilizando-se os testes de Shapiro-Wilk (normalidade dos resíduos) e de Levene (homogeneidade entre as variâncias). Como os resíduos apresentaram distribuição normal e as variâncias foram homogêneas, aplicou-se análise de variância seguida pelo teste de Tukey. Em todos os testes foi utilizado o valor de significância de 0,05.

\section{RESULTADOS E DISCUSSÃO}

Independentemente do método de determinação $\left(70^{\circ} \mathrm{C}\right.$ e $\left.105^{\circ} \mathrm{C}\right)$, o teor médio de água das sementes de E. calycina foi alto, acima de 49,3\%, o que é comum para sementes de espécies de frutos carnosos (Figura 3). Esses altos teores não são particularidade da espécie, mas característica do gênero, presente em sementes de Eugenia handroana D. Legrand (Carvalho et al., 2006), Eugenia brasiliensis Lam., Eugenia crassifolia Kiaersk., E. involucrata, E. pyriformis, Eugenia umbelliflora
O. Berg., E. uniflora (Delgado \& Barbedo, 2007) e Eugenia pleurantha O. Berg. (Masetto et al., 2010).

Exceto para sementes de frutos na coloração vermelha-escura, a disjunção dos intervalos para uma mesma coloração indicou teor de água a $105^{\circ} \mathrm{C}$ superior a $70{ }^{\circ} \mathrm{C}$ (Figura 3 ). A temperatura de $70^{\circ} \mathrm{C}$ pode não ter sido suficiente para remover a água absorvida e adsorvida, mesmo após a estabilização, justificando os valores menores. Em contrapartida, não se pode recomendar a temperatura de $105^{\circ} \mathrm{C}$ porque não há garantias de que apenas a água de constituição tenha sido removida (Campos \& Tillmann, 1996). Como a própria Sociedade Americana de Engenheiros Agrícolas (ASAE, 1992) alerta, há grande diversidade de metodologia oficial para determinação do grau de umidade para uma mesma espécie, não havendo consenso sobre a mais indicada.

Independentemente da temperatura de secagem, houve decréscimo no teor de água à medida que o fruto passou da coloração verde para alaranjada/avermelhada, com teor estabilizado em $49,8 \%$ e $53,7 \%$, quando determinados a $70{ }^{\circ} \mathrm{C}$ e $105{ }^{\circ} \mathrm{C}$, respectivamente (Figura 3). No último estádio de maturação (coloração vermelha-escura), os limites do intervalo de confiança revelaram grandes variações no teor de água no momento da dispersão. Parte dessa variação deve-se à 
mistura de frutos nessa coloração coletados na própria planta, e visivelmente túrgidos, e frutos coletados no solo, visivelmente murchos.

No Experimento I, a coloração do fruto não afetou a capacidade de emergência das plântulas, tampouco tempos inicial, médio e final, velocidade e sincronia (Tabela 2). Percentuais de emergência das plântulas entre $83,7 \%$ e $90,3 \%$ indicaram que as sementes atingiram maturidade fisiológica antes dos primeiros indícios de maturação do fruto, quando ele passa a apresentar coloração alaranjada/avermelhada. No entanto, frutos de E. pyriformis e E. uniflora colhidos com coloração do epicarpo verde não atingiram a maturidade fisiológica (Ávila et al., 2009; Oro et al., 2012).

Não foi particularidade das plântulas de E. calycina apresentar alta capacidade de emergência, entre $80 \%$ e $90 \%$, sem qualquer pré-tratamento das sementes. Para outras espécies do gênero e nas mesmas condições, esse percentual atingiu $87,5 \%$ de plântulas de E. pyriformis (Justo et al., 2007), 90\% em E. stipitata subsp. sororia (Mendes \& Mendonça, 2012), 92\% em E. dysenterica (Duarte et al., 2006) e próximo de 100\% em plântulas

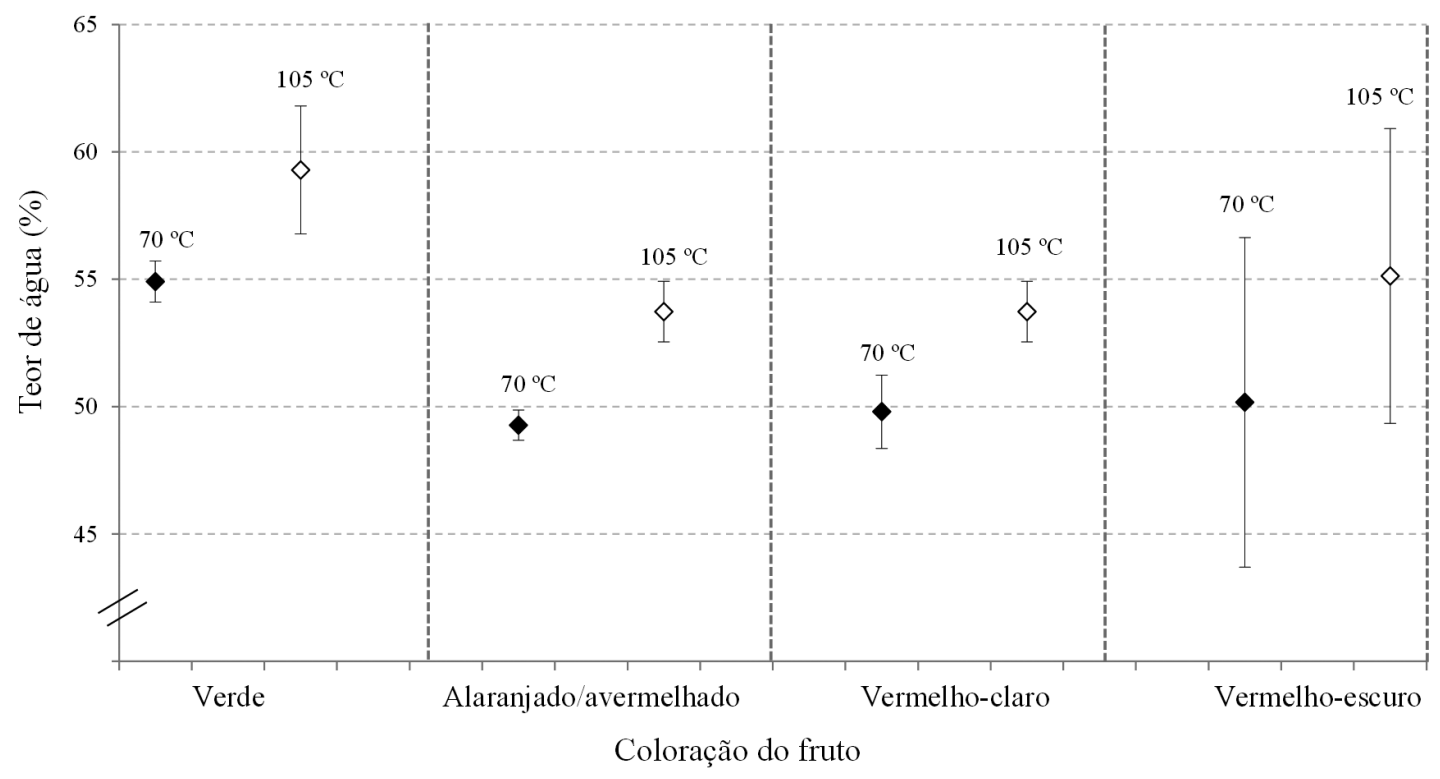

Figura 3. Teor de água de sementes de Eugenia calycina Cambess. advindas de frutos de diferentes colorações. Figure 3. Water content of Eugenia calycina Cambess. seeds, from fruit at different coloring.

Tabela 2. Emergência, medidas de tempo, velocidade e sincronia de emergência de plântulas de Eugenia calycina (pitangueira-do-cerrado) provenientes de frutos com diferentes colorações coletados na Reserva Ecológica do Clube de Caça e Pesca Itororó.

Table 2. Emergence, time measurements, rate and synchrony of Eugenia calycina seedlings from fruits in different coloring, collected in Reserva Ecológica do Clube Caça e Pesca Itororó.

\begin{tabular}{|c|c|c|c|c|c|c|}
\hline $\begin{array}{l}\text { Estádio de maturação } \\
\text { do } \text { fruto }^{1,2} \text { (coloração) }\end{array}$ & $E(\%)$ & $t_{0}$ (dia) & $\bar{t}\left(\mathrm{dia}^{-1}\right)$ & $t_{f}$ (dia) & $\begin{array}{c}V E \\
\left(\text { pl. } \text { dia }^{-1}\right)\end{array}$ & $Z$ \\
\hline Verde & $83,7 \mathrm{a}$ & $23,9 \mathrm{a}$ & $33,3 \mathrm{a}$ & $54,9 \mathrm{a}$ & $0,737 \mathrm{a}$ & $0,053 \mathrm{a}$ \\
\hline Alaranjada/avermelhada & $88,3 \mathrm{a}$ & $24,0 \mathrm{a}$ & $32,7 \mathrm{a}$ & $49,3 \mathrm{a}$ & $0,781 \mathrm{a}$ & $0,068 \mathrm{a}$ \\
\hline Vermelha-clara & $86,2 \mathrm{a}$ & $23,1 \mathrm{a}$ & $32,6 \mathrm{a}$ & $54,0 \mathrm{a}$ & $0,780 \mathrm{a}$ & $0,075 \mathrm{a}$ \\
\hline Vermelha-escura & $90,3 \mathrm{a}$ & $23,3 \mathrm{a}$ & $32,2 \mathrm{a}$ & $49,6 \mathrm{a}$ & $0,819 \mathrm{a}$ & $0,066 \mathrm{a}$ \\
\hline$F{\text { (Levene })^{3}}$ & 0,967 & 1,726 & 0,521 & 0,841 & 0,395 & 0,602 \\
\hline$W$ (Shapiro-Wilk) $^{3}$ & 0,942 & 0,944 & 0,953 & 0,980 & 0,965 & 0,887 \\
\hline
\end{tabular}

${ }^{1}$ Médias seguidas por letras distintas diferem pelo teste de Tukey a 0,05 de significância. ${ }^{2} E$ : percentual de emergência; $t_{o}, \bar{t}$ e $t_{f}$ : tempos inicial, médio e final de emergência. Respectivamente: $V E$ : velocidade de emergência; $Z$ : sincronia. ${ }^{3} F$, $W$ : estatísticas dos testes de Levene e de Shapiro-Wilk: valores em negrito indicam homogeneidade entre as variâncias e resíduos com distribuição normal $(P>0,05)$, respectivamente. 
emergidas de E. brasiliensis, E. involucrata e E. uniflora (Lamarca et al., 2011). Esse potencial de formação de plântulas de Eugenia é ainda maior face à possibilidade do desenvolvimento de novas plântulas a partir de fragmentos de sementes monoembriônicas (Silva et al., 2005; Teixeira \& Barbedo, 2012).

A capacidade de emergência das plântulas de E. calycina contrastou com a emergência inicial (entre 23,1 dias e 24 dias) e final tardia (entre 49,3 dias e 54,9 dias), assim como com tempos médios entre 32,2 dias e 33,3 dias. Em média, menos de uma plântula emergiu por dia ( $V E=0,737$ a 0,819 plântulas dia $^{-1}$; Tabela 2), conferindo ao processo baixa sincronia ( $Z$ entre 0,053 e 0,075$)$.

A emergência lenta e irregular das plântulas e, consequentemente, da germinação, indicou a presença de dormência de origem morfofisiológica das sementes de E. calycina. Com tempo médio de germinação de 30 dias, mecanismos de dormência foram sugeridos para sementes de E. pyriformis (Andrade \& Ferreira, 2000) e de outras espécies da família Myrtaceae, como Campomanesia guazumifolia Cambess., Campomanesia xanthocarpa Mart. ex O. Berg., Eugenia rostrifolia D. Legrand. e Psidium cattleyanum Sabine (Santos et al., 2004). Pela alta germinação, mesmo com tempo médio de 30 dias, a dormência foi descartada para sementes de Myrcia glabra (O. Berg) D. Legrand e Myrcia palustris Barb. Rodr. (Leonhardt et al., 2010).

A igualdade entre médias para todas as características de emergência (capacidade, tempo, velocidade e sincronia) em relação à coloração do fruto também pôde ser comprovada pela similaridade no comportamento das distribuições das frequências relativas (Figura 4). O processo de emergência foi polimodal, distribuído no tempo, típico de sementes com dormência relativa (sensu Labouriau, 1983). Comportamento similar foi verificado para outras espécies do mesmo gênero, como E. stipitata, que mesmo com germinação de 93\% estendeu o processo germinativo para além dos 107 dias, com uma germinação a cada 11 dias (Mendes \& Mendonça, 2012).

As máximas frequências relativas não ultrapassaram $15 \%$ e reforçaram a natureza assíncrona da emergência (Figura 4) como resultado da baixa probabilidade de a

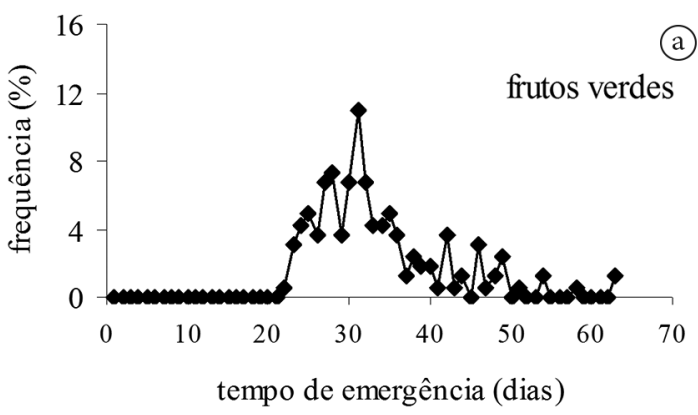

(c)

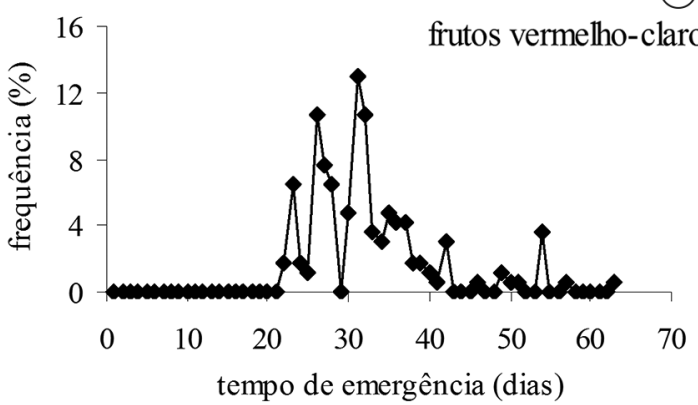

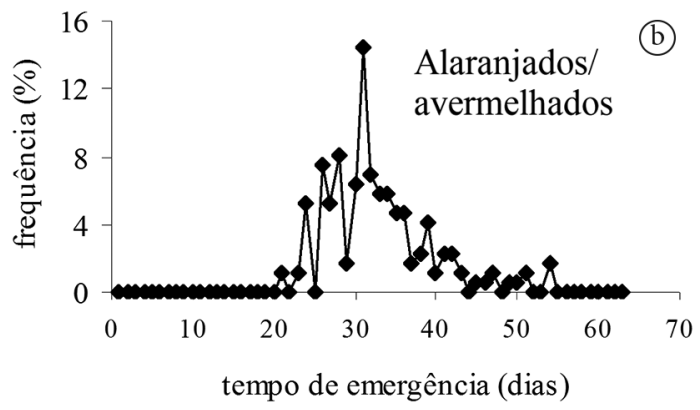

(d)

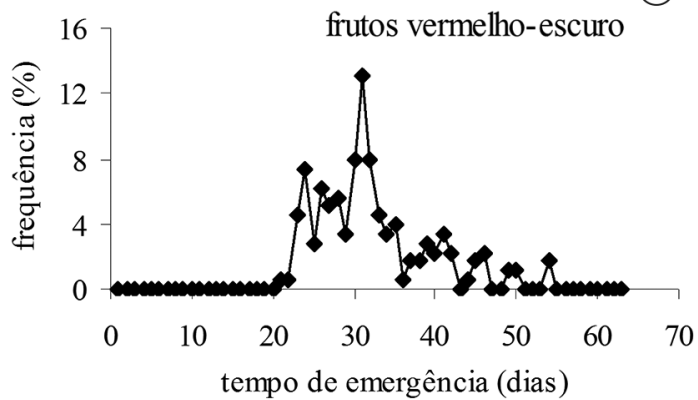

Figura 4. Distribuição de frequência percentual de emergência de plântulas de Eugenia calycina Cambess. advindas de frutos nas colorações (a): verde; (b): alaranjada/avermelhada; (c): vermelha-clara e (d): vermelha-escura coletados na Reserva Ecológica do Clube de Caça e Pesca Itororó.

Figure 4. Percentage frequency distribution of emergence of Eugenia calycina Cambess. seedlings from fruits of (a): green color; (b): orange/reddish; (c): light red and (d): dark red, collected in Reserva Ecológica do Clube Caça e Pesca Itororó. 
emergência ocorrer em um mesmo intervalo de tempo. Isso provavelmente é uma adaptação visando distribuir a germinação no tempo e aumentar a capacidade de sobrevivência no início das chuvas, as quais são sazonais na região do cerrado (Oliveira, 2008).

Assim como para as medidas de emergência de plântulas, a coloração do fruto não afetou significativamente o desenvolvimento das plantas (Experimento II). Plantas de E. calycina não se distinguiram quanto ao incremento em altura $(5,9 \mathrm{~cm}$ a $7,1 \mathrm{~cm})$, comprimento de raiz $(12,1$ $\mathrm{cm}$ e $12,3 \mathrm{~cm})$, diâmetro do caule $(0,08 \mathrm{~cm} \mathrm{e} 0,12 \mathrm{~cm})$ e número de folhas, entre 6,7 e 8,9, ao longo de 210 dias após o transplantio (Tabela 3). Entretanto, plântulas de E. pyriformis atingiram maior comprimento médio de raiz, aumento de massa seca da parte aérea e maior desenvolvimento do sistema radicular quando originadas de frutos no estádio de coloração verde/amarela (Oro et al., 2012).

$\mathrm{O}$ baixo incremento em altura, em comprimento, em diâmetro e no número de folhas ao longo dos 210 dias, mesmo quando a mistura de Plantmax ${ }^{\circledR}$ com Vermiculita ${ }^{\circledR}\left(1: 1 \mathrm{vv}^{-1}\right)$ foi suplementada com o adubo químico $\left(\right.$ Osmocote $\left.{ }^{\circledR}\right)$, revelou crescimento lento das plantas. Há registros também de crescimento lento de plantas de E. dysenterica (Souza et al., 2002; Nietsche et al., 2004), espécie simpátrica a E. calycina.

O substrato comercial (Bioplant ${ }^{\circledR}$ ) suplementado com Vermiculita ${ }^{\circledR}$ e pó-de-coco, na proporção de $20 \%$ e 40\%, respectivamente (Experimento III), também não influenciou no crescimento das plantas de E. calycina (Tabela 4). Uma combinação de fatores pode ser a causa da igualdade entre substratos com composições distintas de material orgânico e estruturante. Embora a Vermiculita ${ }^{\circledR}$

Tabela 3. Incrementos em altura, comprimento de raiz, diâmetro do caule e número de folhas de mudas de Eugenia calycina Cambess. advindas de sementes de frutos de coloração diferente coletados na Reserva Ecológica do Clube de Caça e Pesca Itororó.

Table 3. Increments in height, root length, stem diameter and number of leaves of Eugenia calycina Cambess. seedlings from fruits in different coloring, collected in Reserva Ecológica do Clube Caça e Pesca Itororó.

\begin{tabular}{|ccccc|}
$\begin{array}{c}\text { Estádio de maturação do fruto } \\
\text { (coloração) }\end{array}$ & $\begin{array}{c}\text { Altura } \\
(\mathbf{c m})\end{array}$ & $\begin{array}{c}\text { Comprimento } \\
\text { da raiz }(\mathbf{c m})\end{array}$ & $\begin{array}{c}\text { Diâmetro } \\
\text { do caule }(\mathbf{c m})\end{array}$ & $\begin{array}{c}\text { Número } \\
\text { de folhas }\end{array}$ \\
\hline Verde & $6,18 \mathrm{a}$ & $12,12 \mathrm{a}$ & $0,096 \mathrm{a}$ & $8,93 \mathrm{a}$ \\
\hline Alaranjada/avermelhada & $5,86 \mathrm{a}$ & $12,30 \mathrm{a}$ & $0,081 \mathrm{a}$ & $8,43 \mathrm{a}$ \\
\hline Vermelha-clara & $5,93 \mathrm{a}$ & $12,11 \mathrm{a}$ & $0,120 \mathrm{a}$ & $6,68 \mathrm{a}$ \\
\hline Vermelha-escura & $7,11 \mathrm{a}$ & $12,33 \mathrm{a}$ & $0,120 \mathrm{a}$ & $8,18 \mathrm{a}$ \\
\hline$F($ Levene) & $\mathbf{0 , 2 6 0}$ & $\mathbf{2 , 1 9 7}$ & $\mathbf{0 , 7 4 1}$ & $\mathbf{1 , 5 0 6}$ \\
\hline$W$ (Shapiro-Wilk) $^{2}$ & $\mathbf{0 , 9 7 0}$ & $\mathbf{0 , 9 4 1}$ & $\mathbf{0 , 9 6 9}$ & $\mathbf{0 , 9 7 7}$ \\
\hline
\end{tabular}

${ }^{1}$ Médias seguidas por letras distintas diferem pelo teste de Tukey a 0,05 de significância. ${ }^{2} F$, $W$ : estatísticas dos testes de Levene e de Shapiro-Wilk: valores em negrito indicam homogeneidade entre as variâncias e resíduos com distribuição normal $(P>0,05)$, respectivamente.

Tabela 4. Incrementos em altura, comprimento de raiz, diâmetro do caule e número de folhas de mudas de Eugenia calycina Cambess. submetidas à mistura de substrato comercial, Vermiculita ${ }^{\circledR}$ e pó-de-coco.

Table 4. Increments in height, root length, stem diameter and number of leaves of Eugenia calycina Cambess. seedlings submitted to mixtures of commercial substrate, Vermiculite ${ }^{\circledast}$ and powder-coconut.

\begin{tabular}{|c|c|c|c|c|}
\hline Substrato $^{1}$ & $\begin{array}{c}\text { Altura } \\
(\mathrm{cm})\end{array}$ & $\begin{array}{c}\text { Comprimento } \\
\text { da raiz }(\mathrm{cm})\end{array}$ & $\begin{array}{c}\text { Diâmetro } \\
\text { do caule }(\mathrm{cm})\end{array}$ & $\begin{array}{c}\text { Folhas } \\
\text { (número) }\end{array}$ \\
\hline Bioplant (100\%) & $17,61 \mathrm{a}$ & $18,85 \mathrm{a}$ & $0,291 \mathrm{a}$ & $16,44 \mathrm{a}$ \\
\hline Bioplant $(80 \%)+$ Vermiculita $(20 \%)$ & $17,92 \mathrm{a}$ & $17,90 \mathrm{a}$ & $0,300 \mathrm{a}$ & $24,76 \mathrm{a}$ \\
\hline Bioplant $(80 \%)+$ pó-de-coco $(20 \%)$ & $16,18 \mathrm{a}$ & $17,18 \mathrm{a}$ & $0,326 \mathrm{a}$ & $17,84 \mathrm{a}$ \\
\hline Bioplant $(60 \%)+$ Vermiculita $(40 \%)$ & $17,35 \mathrm{a}$ & $20,45 \mathrm{a}$ & $0,323 \mathrm{a}$ & $19,84 \mathrm{a}$ \\
\hline Bioplant $(60 \%)+$ pó-de-coco $(40 \%)$ & $18,45 \mathrm{a}$ & $19,01 \mathrm{a}$ & $0,355 \mathrm{a}$ & $20,92 \mathrm{a}$ \\
\hline$F(\text { Levene })^{2}$ & 1,333 & 1,586 & 0,194 & 1,103 \\
\hline$W(\text { Shapiro-Wilk })^{2}$ & 0,944 & 0,972 & 0,958 & 0,972 \\
\hline
\end{tabular}

${ }^{1}$ Médias seguidas por letras distintas diferem pelo teste de Tukey a 0,05 de significância. ${ }^{2} F$, $W$ : estatísticas dos testes de Levene e de Shapiro-Wilk; valores em negrito indicam homogeneidade entre as variâncias e resíduos com distribuição normal $(P>0,05)$, respectivamente. 
e o pó-de-coco tenham sido usados com a finalidade de aerar o substrato, ambos têm o inconveniente de reter muita umidade (Carrijo et al., 2002; Martins et al., 2009). Uma análise da plasticidade fenotípica de E. calycina em área de transição cerrado-vereda confirmou a maior densidade populacional no cerrado, indicando a preferência da espécie por solos bem drenados (Cardoso \& Lomônaco, 2003).

Um comparativo com espécies distribuídas nas mesmas áreas de E. calycina também indicou pouco efeito da suplementação de substratos comerciais com material estruturante como areia e casca de arroz carbonizada na produção de mudas de Hancornia speciosa Gomes (Silva et al., 2011) e E. dysenterica (Paiva et al., 2010).

\section{CONCLUSÕES}

A coloração do fruto não influenciou a capacidade de emergência, o tempo inicial, médio e final, a velocidade e a sincronia de emergência das plântulas de E. calycina. O crescimento das plantas até 270 dias de cultivo ocorreu independentemente da coloração do fruto. A Vermiculita ${ }^{\circledR}$ e o pó-de-coco suplementados ao Bioplant ${ }^{\circledR}$ não afetaram o crescimento das plantas até os 120 dias de cultivo.

\section{AGRADECIMENTOS}

Os autores agradecem à CAPES, pela bolsa de Mestrado concedida à primeira autora, e ao Clube Caça e Pesca Itororó de Uberlândia, por possibilitar o acesso às áreas e a coleta dos frutos.

\section{STATUS DA SUBMISSÃO}

Recebido: 24 abr., 2015

Aceito: 27 set., 2015

\section{AUTOR(ES) PARA CORRESPONDÊNCIA}

\section{Vanderley José Pereira}

Instituto de Ciências Agrárias - ICIAG, Universidade Federal de Uberlândia - UFU,

Avenida Amazonas, s/n, Bloco 2E, Sala 01, Umuarama, CP 593, CEP 38400-902, Uberlândia, MG, Brasil

e-mail: vamceres.vanderley@gmail.com

\section{REFERÊNCIAS}

Abreu ND, Mendonça V, Ferreira BG, Teixeira GA, Souza HAD, Ramos JD. Crescimento de mudas de pitangueira (Eugenia uniflora L.) em substratos com utilização de superfosfato simples. Ciência e Agrotecnologia 2005; 29(6): 1117-1124. http://dx.doi.org/10.1590/S141370542005000600003 .

American Society of Agricultural Engineers - ASAE. Moisture measurement unground grain and seeds. 30. ed. Saint Joseph: American Society of Agricultural Engineers; 1992. 404 p.

Andrade RNB, Ferreira AG. Germinação e armazenamento de sementes de uvaia (Eugenia pyriformis Camb.) Myrtaceae. Revista Brasileira de Sementes 2000; 22(2): 118-125. http:// dx.doi.org/10.17801/0101-3122/rbs.v22n2p118-125.

Aquino FG, Walter BMT, Ribeiro JF. Espécies vegetais de uso múltiplo em reservas legais de Cerrado - Balsas, MA. Revista Brasileira de Biociências 2007; 5(1): 147-149.

Ávila ALD, Argenta MDS, Muniz MFB, Poleto I, Blume E. Maturação fisiológica e coleta de sementes de Eugenia uniflora L. (pitanga), Santa Maria, RS. Ciência Florestal 2009; 19(1): 61-68. http://dx.doi.org/10.5902/19805098420.

Borghetti F, Ferreira AG. Interpretação dos resultados de germinação. In: Ferreira AG, Borghetti F. Germinação de sementes: do básico ao aplicado. Porto Alegre: Artmed; 2004. p. 209-224.

Brasil. Regras para análise de sementes. Brasília: Ministério da Agricultura e Reforma Agrária; 2009. 395 p.

Camilo YMV, Souza E, Vera R, Naves RV. Fenologia, produção e precocidade de plantas de Eugenia dysenterica visando melhoramento genético. Revista de Ciências Agrárias (Belém) 2013; 36(2): 192-198.

Campos VC, Tillmann MAA. Comparação entre os métodos oficiais de estufa para determinação do grau de umidade de sementes. Revista Brasileira de Sementes 1996; 18(1): 134-137. http://dx.doi.org/10.17801/01013122/rbs.v18n1p134-137.

Cardoso GL, Lomônaco C. Variações fenotípicas e potencial plástico de Eugenia calycina Cambess. (Myrtaceae) em uma área de transição Cerrado-vereda. Revista Brasileira de Botanica. Brazilian Journal of Botany 2003; 26(1): 131 140. http://dx.doi.org/10.1590/S0100-84042003000100014.

Carrijo AO, Liz RS, Makishima N. Fibra da casca do coco verde como substrato agrícola. Horticultura Brasileira 2002; 20(4): 533-535. http://dx.doi.org/10.1590/S010205362002000400003.

Carvalho LR, Silva EAA, Davide AC. Classificação de sementes florestais quanto ao comportamento no armazenamento. Revista Brasileira de Sementes 2006; 28(2): 15-25. http:// dx.doi.org/10.1590/S0101-31222006000200003. 
Cielo R Fo, Aguiar OT, Baitello JB, Pastore JA, Toniato MTZ, Souza SCPM et al. Aspectos florísticos da Estação Ecológica de Itapeva, SP: uma unidade de conservação no limite meridional do bioma Cerrado. Biota Neotropica 2012; 12(2): 1-20. http://dx.doi.org/10.1590/S167606032012000200015.

Conceição GM, Aragão JG. Diversidade e importância econômica das Myrtaceae do Cerrado, Parque Estadual do Mirador, Maranhão. Scientia Plena 2010; 6(7): 1-8.

Delgado LF, Barbedo CJ. Tolerância à dessecação de sementes de espécies de Eugenia. Pesquisa Agropecuaria Brasileira 2007; 42(2):265-27. http://dx.doi.org/10.1590/ S0100-204X2007000200016.

Duarte EF, Naves RV, Borges JD, Guimarães NNR. Germinação e vigor de sementes de cagaita (Eugenia dysenterica Mart. ex DC.) em função de seu tamanho e tipo de coleta. Pesquisa Agropecuária Tropical 2006; 36(3): 173-179.

Franzon RC, Gonçalves RDS, Antunes LEC, Raseira MDCB. Vegetative propagation of surinam cherry (Eugenia uniflora L.) genotypes from Southern Brazil, through cleft graft. Revista Brasileira de Fruticultura 2010; 32(1): 262-267. http://dx.doi.org/10.1590/S0100-29452010005000003.

Gomes CC, Moura TM. Estrutura genética em população de plantas do Cerrado. Revista Agrotecnologia 2010; 1(1): 33-52. http://dx.doi.org/10.12971/2179-5959.v01n01a03.

Justo CF, Alvarenga AD, Alves E, Guimarães RM, Strassburg RC. Efeito da secagem, do armazenamento e da germinação sobre a micromorfologia de sementes de Eugenia pyriformis Camb. Acta Botanica Brasílica 2007; 21(3): 539-551. http://dx.doi.org/10.1590/S010233062007000300004 .

Labouriau LG. A germinação das sementes. Washington: Organização dos Estados Americanos; 1983. 174 p. Série de Biologia.

Lamarca EV, Silva CV, Barbedo CJ. Limites térmicos para a germinação em função da origem de sementes de espécie de Eugenia (Myrtaceae) nativas do Brasil. Acta Botanica Brasílica 2011; 25(2): 293-300. http://dx.doi. org/10.1590/S0102-33062011000200005.

Landrum LR, Kawasaki ML. The genera of Myrtacae in Brazil: an illustrated synoptic treatment and identification keys. Brittonia 1997; 49(4): 508-536. http://dx.doi. org/10.2307/2807742.

Leonhardt C, Calil AC, Fior CS. Germinação de sementes de Myrcia glabra (O. Berg) D. Legrand e Myrcia palustris DC. - Myrtaceae armazenadas em câmara fria. Iheringia Série Botânica 2010; 65(1): 25-33.

Maguire JD. Speed of germination-aid in selection and evaluation for seedling emergence and vigor. Crop Science 1962; 2(2): 176-177. http://dx.doi.org/10.2135/cropsci19 62.0011183X000200020033x.
Martins CC, Bovi MLA, Spiering SH. Umedecimento do substrato na emergência e vigor de plântulas de pupunheira. Revista Brasileira de Fruticultura 2009; 31(1): 224-230. http://dx.doi.org/10.1590/S0100-29452009000100031.

Masetto TE, Davide AC, Faria JMR, Silva EAA, Rezende RKS. Avaliação da qualidade de sementes de Eugenia pleurantha (Myrtaceae) pelos testes de germinação e tetrazólio. Agrarian 2010; 2(5): 33-46.

Medeiros LF, Costa FC, Curi PN, Moura PHA, Tadeu MH. Diferentes substratos na produção de mudas de uvaieira (Eugenia pyriformis Cambess.). Revista Verde 2010; 5(2): 209-212.

Mendes AMS, Mendonça MS. Tratamentos pré-germinativos em sementes de araçá-boi (Eugenia stipitata). Revista Brasileira de Fruticultura 2012; 34(3): 921-929. http:// dx.doi.org/10.1590/S0100-29452012000300035.

Nietsche S, Gonçalves VD, Pereira MCT, Santos FA, Abreu SD, Mota WF. Tamanho da semente e substratos na germinação e crescimento inicial de mudas de cagaiteira. Ciência e Agrotecnologia 2004; 28(6): 1321-1325. http:// dx.doi.org/10.1590/S1413-70542004000600014.

Oliveira EAM. Fenologia e biologia reprodutiva de espécies de Cerrado. In: Sano SM, Almeida SP, Ribeiro JF, editores. Cerrado: ecologia e flora. Brasília: Embrapa Informação Tecnológica; 2008.

Oro P, Schulz DG, Volkweis CR, Bandeira KR, Malavasi UC, Malavasi MM. Maturação fisiológica de sementes de Eugenia pyriformis Cambess. e Eugenia involucrata DC. Biotemas 2012; 25(3): 11-18. http://dx.doi.org/10.5007/21757925.2012v25n3p11.

Paiva SP So, Luz PB, Silveira TLS, Ramos DT, Neves LG, Barelli MAA. Substratos na produção de mudas de três espécies arbóreas do cerrado. Revista Brasileira de Ciências Agrárias 2010; 5(2): 238-243. http://dx.doi.org/10.5039/ agraria.v5i2a741.

Pereira ME, Pasquelete A. Desenvolvimento sustentável com ênfase em frutíferas do Cerrado. Estudos 2011; 38(2): 333-363.

Ranal MA, Santana DG. How and why to measure the germination process? Revista Brasileira de Botanica. Brazilian Journal of Botany 2006; 29(1): 1-11. http://dx.doi. org/10.1590/S0100-84042006000100002.

Ribeiro RA, Rodrigues FM. Genética da conservação em espécies vegetais do Cerrado. Revista de Ciências Médicas e Biológicas 2006; 5(3): 253-260.

Santos CMR, Ferreira AG, Áquila MEA. Características de frutos e germinação de sementes de seis espécies de Myrtaceae nativas do Rio Grande do Sul. Ciência Florestal 2004; 14(2): 13-20.

Scalon SPQ, Neves EMS, Maseto TE, Pereira ZV. Sensibilidade à dessecação e ao armazenamento em sementes de Eugenia pyriformis Cambess. (uvaia). Revista 
Brasileira de Fruticultura 2012; 34(1): 269-276. http:// dx.doi.org/10.1590/S0100-29452012000100036.

Sena LHDM, Matos VP, Sales A, Ferreira E, Pacheco MV. Qualidade fisiológica de sementes de pitangueira submetidas a diferentes procedimentos de secagem e substratos - Parte 1. Revista Brasileira de Engenharia Agrícola e Ambiental 2010; 14(4): 405-411. http://dx.doi. org/10.1590/S1415-43662010000400009.

Silva CV, Bilia DAC, Barbedo CJ. Fracionamento e germinação de sementes de eugenia. Revista Brasileira de Sementes 2005; 27(1): 86-92. http://dx.doi.org/10.1590/ S0101-31222005000100011.

Silva EA, Oliveira AC, Mendonça V, Soares FM. Substratos na produção de mudas de mangabeira em tubetes. Pesquisa Agropecuária Tropical 2011; 41(2): 279-285.

Souza ERB, Naves RV, Oliveira MF. Initial fruiting of the cagaita tree (Eugenia dysenterica DC) cultivated in Goiânia, Goiás, Brazil. Revista Brasileira de Fruticultura
2013; 35(3): 906-909. http://dx.doi.org/10.1590/S010029452013000300030.

Souza ERBD, Naves RV, Carneiro IF, Leandro WM, Borges JD. Crescimento e sobrevivência de mudas de cagaiteira (Eugenia dysenterica DC) nas condições de cerrado. Revista Brasileira de Fruticultura 2002; 24(2): 491-495. http://dx.doi.org/10.1590/S0100-29452002000200042.

Teixeira CC, Barbedo CJ. The development of seedlings from fragments of monoembryonic seeds as an important survival strategy for Eugenia (Myrtaceae) tree species. Trees (Berlin) 2012; 26(3): 1069-1077. http://dx.doi. org/10.1007/s00468-011-0648-5.

Tonetto TS, Prado AP, Araujo MM, Sccoti MSV, Franco ETH. Dinâmica populacional e produção de sementes de Eugenia involucrata na Floresta Estacional Subtropical. Floresta e Ambiente 2013; 20(1): 62-69. http://dx.doi. org/10.4322/floram.2012.072. 